\title{
Information theory based scoring function for predicting protein-ligand binding affinity Mahesh Kulharia
}

\author{
Address: MPI für molekulare Physiologie, 44227 Dortmund, Germany \\ from 3rd German Conference on Chemoinformatics \\ Goslar, Germany. II-I3 November 2007 \\ Published: 26 March 2008 \\ Chemistry Central Journal 2008, 2(Suppl I):P44 doi:I0.II86/I752-I53X-2-SI-P44
}

This abstract is available from: http://www.journal.chemistrycentral.com/content/2/SI/P44

(c) 2008 Kulharia

The development and validation of a new knowledge based scoring function (SIScoreJE) to predict binding affinity between proteins and ligands is presented. SIScoreJE efficiently predicts the binding energy between a small molecule and its protein receptor. Protein-ligand atomic contact information was derived from a "nonredundant dataset" (NRD) of over 3000 X-ray crystal structures of protein-ligand complexes. This information was classified for the individual "atom contact pairs" (ACP) according to a number of current atom-type classification schemes in addition to two of our own before calculating the atomic contact preferences. The preferences were calculated using an information theoretic relationship of joint entropy. Among 18 different atom-type classification schemes "ScoreJE atom type set2" (SATs2) was found to be most suitable for our approach. Single-body solvation potentials (SSP) were derived from the atomic contacts between the protein atom types and water molecules modelled using AQUARIUS2. Validation was carried out using an evaluation dataset of 100 protein-ligand complexes with known binding affinities. A combined SSP/ ScoreJE (SIScoreJE) performed significantly better than ScoreJE alone. Also SIScoreJE and ScoreJE performed better than GOLD::GoldScore, GOLD::ChemScore, and XScore. 